\title{
A THEORY OF HUMAN ECOLOGICAL CONTROL NETWORKS: THE ROLE OF AFFORDATION, EMOTION AND INTUITION IN THE DESIGN OF HUMAN ENVIRONMENTS
}

\author{
Waldemar Karwowski \\ Center for Industrial Ergonomics \\ University of Louisville \\ Louisville, KY 40292, U.S.A. \\ karwowski@louisville.edu \\ and \\ Krystyna Gielo-Perczak \\ Liberty Mutual Research Center for Safety \& Health \\ 71 Frankland Road \\ Hopkinton, MA 01748, U.S.A.
}

Keywords: Human environment, brain, behavior, control, affordance, affordation, emotion, intuition, learning, adaptation, tuning, theory, human ecological control networks (HECN)

\begin{abstract}
This paper introduces a theory of the human ecological control networks (HECON). The proposed theory synthesizes the selected concepts from the psychological theory of affordances, cybernetics, ergonomics design, and neuroscience. Affordances of the environment are interrelated with the personal human attributes in terms of neural (brain) manifestations of emotions and intuition. A process of ecological perception and cognition, i.e. the human understanding of the environment in terms of what the environment affords to the operator and the acting upon such an understanding in order to exercise control over that environment (workplace design) was defined as affordation. The affordation-, emotion- and intuition-based design models for human environments correspond to the learning-, adaptiveand tuning-based human ecological control networks, respectively.
\end{abstract}

\section{Introduction}

The complexity of mutual relationships between human operators and their (work) environments often demands an effective human control, predictions and decisions to be made in the presence of uncertainties and unforeseen changes in work systems parameters (Zadeh, 1973; Gibson, 1979, Karwowski, 1991; Karwowski 1992; Karwowski et al, 1999; Karwowski, 2000).

As discussed by Gielo-Perczak and Karwowski (2002), the description of human operators who actively participate in purposeful work tasks in a given environment, and their performance on such tasks should also reflect the complexity of the human brain activity, which includes cognition and the dynamic processes of knowing.

A classical way in which human abilities at work can be categorized is by applying the cognitive engineering framework proposed twenty years ago by Rasmussen
(1983). This approach includes the skill-based, rulebased, and knowledge-based modes of human performance, with reference to different classes of human information processing that involves signals, signs and symbols, respectively.

Skill-based behavior refers to sensory-motorperformance which takes place without conscious control, as smooth, automated, and highly integrated patterns of behavior. In this view, human activities are considered as a sequence of skilled acts composed for the actual situation. Rule-based behavior refers to goaloriented performance structured by feed-forward control through a stored rule or procedure allowing composition of a sequence of subroutines in a familiar work situation. Finally, knowledge-based behavior refers to goal-controlled performance, where the goal is explicitly formulated based on knowledge of the environment and the aims of the person. 
The above cognitive engineering approach to the modeling of human behavior based on the classical stimulus-response paradigm of information processing, with human performance as mapping from a set of stimuli to a set of responses. However, as the human brain is a dynamical system that aims to exercise control over the environment, the design of human can be modeled as a dynamic, nonlinear process taking place over the interactions between the human brain and the environment, based on the concepts of affordances, emotion and intuition.

The interrelationships between affordances, emotion and intuition play an important role and should be taken into account in design of human work environments.

\section{Objectives}

The three models of human performance proposed by Rasmussen (1983), stipulate that people can form internal representations that constitute their structural and functional understanding of a work system, and which allow them to describe, explain, understand, and predict the work system behavior.

As departure from the above categorization scheme for human performance, in this paper we propose an expanded and supplementary framework, based on dynamic human functioning afforded by environment. Such a framework is defined as mapping from a set of affordances, emotions, and intuition to human learning, adaptive- and tuning-based performance. In the context of design of human environment, we have named these mapping processes the human ecological control networks (HECON).

\section{A neuro-ecological design approach based on affordation, emotion and intuition}

Human performance is manifested in several brain activities, including thought, perception, emotion, will, memory, and imagination (Parasuraman 2000).

Therefore, we also need tools for design of human environment that take into account human emotions, imagination and intuition in reference to affordances of the environment.

\subsection{The theory of affordances}

The noun affordance was first introduced by Gibson (1979) to underscore the complementarity of the human and the environment. The affordances of the environment are what it offers or provides the human (and other organisms), for human benefit or human ill. According to Gibson (1986) what we perceive when we look at objects are their affordances, not their qualities, i.e. what the objects afford us is what we normally pay attention to. Humans aim to change and do change the environment in order to change what the environment affords them.

Affordances are opportunities for action for a particular organism. Thus, an affordance is objective in the sense that it is fully specified by externally observable physical reality, but subjective in the sense of being dependent on the behavior of a particular kind of organism. Gibson (1979) suggested that perception of the world is based upon perception of affordances, of recognizing the features of the environment, which specify behaviourally relevant interaction. An animal's ecological niche is defined by what its habitat affords. When an animal's physiological state no longer meets its internal demands, action is generated to bring it to a more satisfying state.

In view of the above, there is no need to assume the presence of a complete internal representation of the external world; when environmental regularities are allowed to take part in behavior, they can give it coherence without the need of explicit internal mechanisms for binding perceptual entities. The question is: what kind of information processing does the brain do and what is its purpose. The answer is that the brain is exerting control over its environment. It does so by constructing behavioral control networks, which functionally extend outside of the body, making use of consistent properties of the environment including the behavior of other organisms. These networks and the control they allow are the very reason for having a brain. To understand them, we must move beyond the input-output processing emphasized by computational models of human performance.

\subsection{Affordation}

An affordance is both an objective and subjective property of the environment and the human operator (Gibson (1986). Affordances are relative to the characteristics of the individual in terms of a human operator's limitations and capacities. Affordance must have characteristics that are perceivable by the human operator, that is, must have meaning. From the systems dynamics point of view, the affordances act as attractors in system behavior towards which the human operator gravitates with regard to her/is intentions, needs, or requirements and goals.

According to Gibson (1986), the perceiving of an affordance is a process of receiving an ecological value that affords something of interest to a human.

Following the above, we are hereby prose the new term, affordation, to define a process of ecological perception and cognition, i.e. the human understanding of the environment in terms of what the environment affords to the operator, and the acting such an upon understanding in order to exercise control over that environment. In view of the above, ergonomics design 
is an attempt of affordation control, i.e. the process of changing what the environment affords to us. Humans attempt to change what their environment affords to them.

\subsection{Cybernetics: human brain as the control network}

The notion of behavior as control is one of the fundamental concepts of cybernetics (Wiener 1958). Ashby (1965), in his seminal work entitled Design for a Brain: The Origins of Adaptive Behavior, outlined a theory of control systems capable of maintaining homeostasis and adapting their sensory motor architecture through elementary learning processes. As discussed by Cisek (1999), the feedback nature of behavior also has strong roots in psychology. For example, the research on sensorimotor development by Jean Piaget was influenced by cybernetics. Powers (1973) proposed a model of a behavioural control hierarchy applicable to simple reflexes and complex social interactions, leading to development of the perceptual control theory (Bourbon 1995).

Cisek (1995) postulated that the function of the brain is to assert control over the organisms' state within its environment. Furthermore, he argued that the description of behavior, as the computation of a response to a stimulus is incomplete because it only examines what occurs between the reception of stimulus information and the generation of an action. Therefore, the behavior should be described as a control process where actions are performed in order to affect perceptions. Such an approach allows explaining how internal representations, even symbols, can have meaning for an organism, and how actions can be motivated by organic needs (Cisek 1999).

Viewing the brain as a control system, rather than an input-output device, stipulates shifting from emphasis on representations to emphasis on behavior, and from the analysis of serial stages of information processing to analysis of parallel control streams (Cisek 1999), allowing the correction of two important misconceptions: 1) severing the behaving organism from its environment; and 2) decomposing behavior into perception, cognition, and action modules which are then studied in isolation.

The brain, which operates with large numbers of noisy elements, working in parallel computing units and distributed memory, processes information for the purpose of exerting control over its environment (Cisek 1999). Following this view, we propose to describe human performance in terms of the dynamic, nonlinear, teleological connective networks that express the human brain purpose of ecological control, called here collectively as the human ecological control networks (HECON).
3.4. Axiomatic approach to ecological design In this view, the purpose of ergonomics is to optimize the beneficial affordances of the environment in order to ensure control of the environment by the human operator. Ergonomics design is the design of affordances provided by the artifacts that are compatible with human needs and requirements (Karwowski, Marek and Noworol, 1988; Karwowski, 1991; Karwowski, 1992; Karwowski, Marek and Noworol, 1994; Karwowski et al, 1999; Karwowski 2000).

The best design is one that maximizes desirable (positive) affordances of the environment and minimizes the undesirable (negative) affordances of the environment. This concept was discovered and confirmed by a study on the golden section in workplace/tool design as a preferred design, harmonizing an individual's body dimensions with the work environment (Gielo-Perczak, 2001).

\subsection{Ecological approach to design of human environments}

As behavior affords behavior, environment affords performance and performance affords performance. According to Turvey and Shaw (1999), from an ecological perspective the classical distinction between perceptual and conceptual needs to be realized. Affordances and their specifications are counterpoints to the view that the meanings-constraining-humanbehavior reside in the brain. A prevalent presumption of cognitivism is that knowledge in the form of concepts of denumerable static objects and relations is needed for an animal to behave felicitously (e.g., Nilsson 1991).

Affordances contradict this presumption. As an animal moves with respect to surrounding substances, surfaces, places, etc., some opportunities for action persist, some newly arise, and some dissolve, even though the surroundings analyzed formally as conceptualized objects and relations remain the same. A change of pace or a change of location can mean that a cleft in the ground now affords leaping over whereas at an earlier pace or location it did not (Turvey and Shaw 1995). Further, subtle changes of action can give rise to multiple and marked variations in the opportunities for subsequent actions. The environment-for-the-animal is dynamic and action oriented while the environment-initself, that which has been the target of cognitive modeling by means of concepts, is fixed and neural with respect to an animal and its actions (Kirsch 1991a).

A rethinking of the conceptual versus perceptual distinction in terms of invariance detection is encouraged by arguments for the symmetry and 
specificity of animal-environment systems. Detecting invariants is at the heart of what an animal knows about its environment. There are two kinds of invariant detection, which are different but complementary. The kind of detection that underlies the perception of persisting substances, surfaces, places, is more fundamental than the kind of detection that underlines the perception of similarities. Gibson suggested the term resonance of perceptual systems and the term abstraction by perceptual systems for the kind of invariance that relates to classes and categories. "The real progress of explaining peculiarly human instances of knowing about will have to await an understanding of the symmetry and specificity that fix the directness of perceiving as a property of all animal-environment systems."

\section{Design for human emotions}

The rapid development of neurobiology and neuroscience has led to a greater understanding of biological cognitive functioning (Levine 2000). Emotions are integrated in a hierarchy of neurological processes that influence brain perceptual functions. There is behavioral and physiological evidence for the integration of perception and cognition with emotion. An emotion defines the organism's dynamic structural pattern and their interactions may lead to specific responses in a work system (environment). Emotions as mental states represent responsiveness (fear, pleasure, anger) and, along with sensation and perception, cognition, personal and social context, are modified by memory (Stuss and Alexander 2000). Emotions, and the feelings that follow emotions, are an integral part of the value systems necessary for laying down long-term memory and for reasoning and conscious decision-making. Moss and Damasio (2001) noted that without emotion we cannot understand how the organism maintains homeostasis in the face of environmental challenges. Damasio (1994) observed without considering emotion, we cannot place the mind in relation to its revolutionary origins, nor to its role relative to the organism as a whole.

As discussed by Gielo-Perczak and Karwowski (2002), humans possess emotions and it is crucial how they activate and transpose them to a work environment. We need to determine the influence of emotions on the phenomenon of out-of-logic mental activity, taking into account individual differences to which people depend on the experiential and rational thought processing. Emotions are integrated in a hierarchy of neural brain operations from arousal and drive to perceptual processes to self-awareness. Anatomical evidence converges to illuminate the structure of the progressive integration of perception and cognition with emotion. Essential brain structures for the perception, expression and developmental evolution of awareness include the hypothalamus, amygdala, and sensory association cortices (particularly of the right hemisphere) and the frontal lobes (Stuss and Alexander 2000). Functional models of emotion are intended to reveal the adaptive function of emotion. Emotions contribute to an adaptive value for the organism by contradicting the convention that emotion is not rational (Moffat and Frijda 2000). Emotions are concern-activated response patterns which take control in order to prepare the organism for a suitable change in action. A concern can be the thought of a desired state or event.

\section{Design for human intuition}

Intuition, or a concept of tacit knowledge, refers to information that cannot easily be communicated verbally. Intuition to the ancients was fire, but more in the sense of a spark that initiates; it is the creative, the divine spark, the spirit itself (Young, 1976). For an intuitive person, free decision is effective as an opposite in nature to the predictable type of decision. Intuition responds to its own philosophy of the future, but it does not react in conformity to the laws that govern and contain secular interchange (Young, 1976). Intuition guesses the exceptional and it does not work by rules. Intuitions are often things learned from direct experience and training.

As dicussed by Gielo-Perczak and Karwowski (2002), intuition has close links with perception and underlying assumptions about perception. By examining intuition through an ecological psychology framework, the problem takes on a different character which is no longer focused solely on the expert's cognitive (or perceptual) processes. What other researchers have often called 'intuition' is what Gibson (1986) termed 'direct perception'. Although some aspects of intuition may be hard-wired through evolution, intuition as direct perception is information-based and can be developed through education and extensive, deliberate practice. Characterized as direct perception, intuition is an observable, lawful phenomenon that is measurable and potentially teachable.

Intuition can be analyzed as groups of: situational factors, process characteristics, and product. The situational factors of intuition refer to the factors that must be in place in order for intuition to occur, namely knowledge and experience, empathy, as well as incomplete data and uncertain situations. The second group of intuition characteristics has to do with the process of intuition, which is more interpretive, holistic and goal-directed. The third category of characteristics has to do with intuition as a product which includes problem-solving and the rational justification of intuition by means of reflection. 
Intuition is an internal experience. It is a way of knowing something directly without an intervening analytic thought. Intuition adds value when creativity and innovation are necessary. Intuition is the skill of responding appropriately, in the moment, to specific situations. Therefore, it is important to consider intuition as a component of adaptive function, and propose a neuro-ecological approach to human performance. Furthermore, intuition should be enhanced in the workplace, becoming familiar with some of the ways in which experienced, and facilitating co-workers to express their intuitions more openly. There is a need to blend a theoretical and tacit knowledge.

\section{The neuro-ecological models for design of human environments}

Following the ecological framework of theory of affordances and the neuroscience perspective of humans at work, we propose to consider a human system as an interconnected control network of distinctions, including affordation, emotion and intuition. These components are constantly interacting with the human brain and the musculoskeletal system in a network which allows a system to respond to specific affordances (or changes in affordances, i.e. disturbances) in the control of work environment. The entire neuro-ecological network responds to changing affordances (selected disturbances) by rearranging its patterns of connectivity. Specific changes in affordances can trigger specific structural changes in the connectivity throughout the network.

In order to describe the neuro-ecological network connectivity there is a need to model the mutual relationships between affordation, perception, emotion and intuition. These specific relationships allow defining three basic models of human ecological control (for more information see Gielo-Perczak and Karwowski, 2002). A network with perception in addition to cognition and body sensors is considered as a learning type. A network possessing all distinctions of a learning type plus emotion is considered as an adaptive type, and an adaptive network type with intuition is considered as a tuning type (Fig.1).

The proposed network types can be represented analytically in terms of control-theoretical models for quantitative predictions in the various work (environment) behaviors (activities). As discussed by Gielo-Perczak and Karwowski (2002), the above concerns open a space of possibilities in which the interrelationship between brain and cognitive science, neuroscience, dynamic systems modeling, control of engineering systems and human rationality can foster the value of affordation, emotion and intuition in human performance.

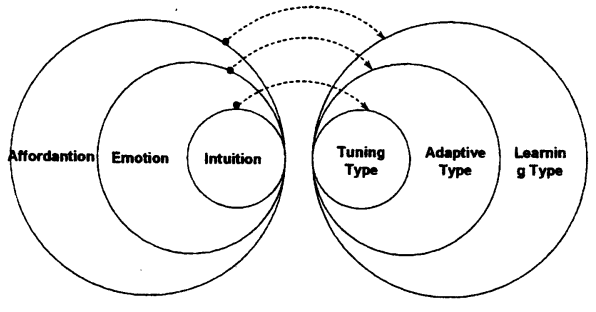

Fig. 1. Relationships between affordation, emotion and intuition in neuro-ecological modeling.

\subsection{Learning-based ecological control network}

The design of a learning-based control network involves the attributes of affordation, cognition, and body sensors, which collectively determine the control of environment (i.e. work process). The information pick-up is considered as a single-channel mechanism (Fig.2). In this model, three phases are considered: 1) conversion of the input affordance of a work process to sensory output by the body sensors, 2) perception process, 3) cognition decision and response selection.

The proposed ecological control network utilizes body sensors' feedback loop together with the loop which continually adds the perception and cognition parameters in an attempt to make the decision output as safe as possible. Feedback control is based on using the outcome of the task in order to control it. It uses the difference between the actual output $y$ and the input $x$ in order to reduce it.

The basic affordances of the environment are perceivable directly. One major advantage of the feedback control is sensitivity to changes in the parameters of the task. A learning type must have a particular response to a specific input signal by repeating the instructions. Learning involves a process of forcing a human with precise action to control (change) the environment, i.e. the work process.

\subsection{Adaptive-based ecological network} Adaptation is considered to be a process of modifying the parameters of the system and the control actions (Fig. 2). An adaptive-based ecological control network continuously searches for the optimum within its allowed environment (work system) possibilities by an orderly trial-and-error process and can resolve a problem according to the available information being utilized in the on-line execution of the control. 


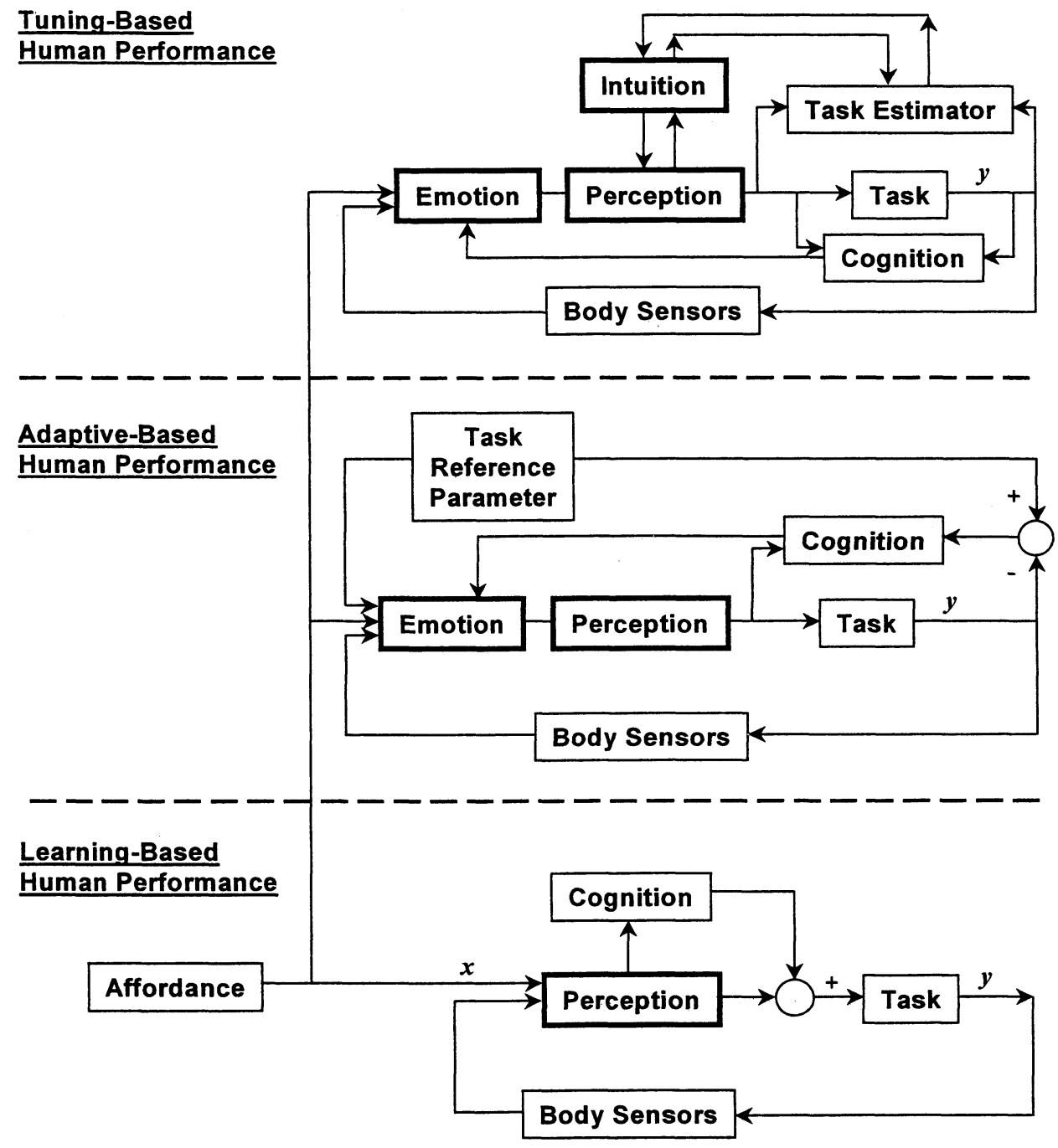

Fig. 2. A general framework of the human ecological control networks.

As discussed by Gielo-Perczak and Karwowski (2002), an important element of adaptive control is the learning of drifting parameters of the environment (work process). As the work process unfolds, additional information becomes available either accidentally, through past control actions, or as a result of active probing which itself is possible by control action. This can be done by anticipating how future estimation will be beneficial to the control objective. An adaptive ecological network utilizes, in addition to the available information at the time, the knowledge that future observations will be made and regulates its adaptation or learning accordingly. The network considers the specifications of the reference parameters of the task, 
which determine the desired ideal response of the work process output to the command signal. The network consists of a feedback control loop with body sensors with an outer loop which continually adjusts the perception parameters in an attempt to make the decision output the same as the reference parameters of the task output. The adjustment control capabilities of an adaptive type make an error of controlling as close to zero as possible.

\subsection{Tuning-based ecological network}

The tuning-based ecological network represents a subgroup of adaptive types of ecological control networks; they can easy effectuate and implement complex tasks. They can control complex processes with a wide variety of work tasks presented by characteristics involving unknown parameters like time delay, time-varying work process dynamics and stochastic disturbances.

The tuning network can control unknown tasks using recursively-estimated values of the work parameters. The analytical control technique of the tuning network consists of three main steps: 1) identification of a work process, 2) control during each sample interval, and 3) drive itself toward the control optimum by making a proper modification.

The tuning-based network, therefore, has the ability to tune itself initially and to re-tune. The tuning system consists of two feedback loops with the body sensors and intuition. Through the outer loop with intuition, a tuning type continually adjusts the task parameters (Fig.2). This approach to tuning control is an ambiguous control process itself. In some tuning controls it is possible to re-parameterize the work process (i.e. environment) such that it can be expressed in terms of the work-controlled parameters.

In terms of Gibson's ecological framework (1986), a tuning-based control network resonates in response to a range or frequency of information which is more or less close to its own knowledge. The maximum response occurs only when the range or frequency of information coincides with its own. The resonance may be more or less finely 'tuned', occurring when a knowledge of past and present systems are most closely similar (Sheldrake, 1981).

Generally, a human ecological network is referred to as a control system when it is capable of learning. Thus, in all types of ecological networks (learning, adaptive and tuning) there exists feedback (control) through body sensors. Since the feedback is used as input for the learning processes, the human ecological control networks can exhibit improved performance over time. However, for adaptive and tuning control networks, there are feed forward controllers through cognition and intuition which help them to adapt to a reference parameter of the task or tune to a task estimator of the environment (i.e. work system). For adaptive and tuning ecological control networks, the information pickup is considered a multiple input due to the complex nature of emotions.

\section{Conclusions}

In this paper, we proposed a framework for the human ecological control networks (HECON) by combining the ecological approach (affordances) with the neural (brain) manifestations of the internal human attributes (emotions and intuition) that are critical in exercising control over the environment. Neural networks with a principle of organization were applied to design of human environment. The principle of organization in personality was evaluated and confirmed in studies by McDowell (2001). The affordation, emotion, and intuition-based framework is a taxonomy that can be used to characterize various types of human ecological control networks.

\section{References}

[1] R. Ashby: Design for a Brain: The Origin of Adaptive Behavior, London: Chapman and Hall, 1965. [2] P. Cisek: Beyond the Computer Metaphor: Behavior as Interaction, in: Nunez, R. and Freeman, W. J. (Eds), Reclaiming Cognition: The Primacy of Action, Intention and Emotion, United Kingdom: Imprint Academic, pp. 125-142, 2000.

[3] A.R. Damasio: Descartes' Error: Emotion, Reason, and the Human Brain, New York: Oxford University Press, 1994.

[4] J. J. Gibson: The Ecological Approach to Visual Perception, Hillsdale, NJ: Lawrence Erlbaum Associates, Publishers, 1986.

[5] K. Gielo-Perczak: The golden section as a harmonizing feature of human dimensions and workplace design, Theoretical Issues in Ergonomics Science, 2(4), 335-350, 2001.

[6] Gielo-Perczak, K. and Karwowski, W., 2002, A neuro-ecological models of human performance based on affordance, emotion and intuition, Ergonomics (submitted).

[7] W. Karwowski: Complexity, fuzziness and ergonomic incompatibility issues in the control of dynamic work environments, Ergonomics, 34 (6), 671$686,1991$.

[8] W. Karwowski: The human world of fuzziness, human entropy, and the need for general fuzzy systems theory, Journal of Japan Society for Fuzzy Theory and Systems, 4 (5), 591-609, 1992.

[9] W. Karwowski, T. Marek, and C. Noworol: The complexity-incompatibility principle in the science of ergonomics, in F. Aghazadeh (ed), Advances in Industrial Ergonomics \& Safety VI, London: Taylor \& Francis, 37-40, 1994. 
[10] W. Karwowski: Symvatology: The science of an artifact-human compatibility, Theoretical Issues in Ergonomics Science, 1, 2000.

[11] W. Karwowski, J. Grobelny, Y. Yang, and W. G. Lee: Applications of fuzzy systems in human factors, in H. Zimmerman (ed), Handbook of Fuzzy Sets and Possibility Theory, Boston, MA: Kluwer Academic Publishers, 589-620, 1999.

[12] W. Karwowski, W. G. Lee, B. Jamaldin, P. Gaddie, and R. Jang: Beyond psychophysics: A need for cognitive modeling approach to setting limits in manual lifting tasks, Ergonomics, 42 (1), 40-60, 1999.

[13] D. S. Levine: Introduction to Neural and Cognitive Modeling, 2nd edition Mahwah, NJ: Lawrence Erlbaum Associates, 2000.

[14] M. J. McDowell: Principle of organization: a dynamic-systems view of the archetype-as-such, Journal of Analytical Psychology, 46, 637-654, 2001. [15] D. C. Moffat and N. H. Frijda: Functional models of emotion, in G. Hatano, N. Okada, and H. Tanabe (eds.), Affective Minds, Amsterdam: Elsevier Science, 59-68, 2000.

[16] H.Moss and A. R. Damasio: Emotion, cognition, and the human brain. Annals of the New York Academy of Sciences, 935, 98-100, 2001.

[17] R. Parasuraman (Ed.): The Attentive Brain, Cambridge: The MIT Press, 2000.
[18] W. Powers: Behavior: The Control of Perception, New York: Aldine Publishing Company, 1973.

[19] J. Rasmussen: Skills, rules, and knowledge; signals, signs, and symbols, and other distinctions in human performance models, IEEE Transactions on Systems, Man, and Cybernetics-Part C: Applications and Reviews, SMC-13, 257-266, 1983.

[20] J. Rasmussen: A catalogue of models, Information Processing and Human-Machine Interaction: An Approach to Cognitive Engineering, New York: NorthHolland, 171-183, 1986.

[21] D. T. Stuss and M. P. Alexander: The anatomical basis of affective behavior, emotion and selfawareness: a specific role of the right frontal lobe, in G. Hatano, N. Okada and H. Tanabe (eds), Affective Minds, Amsterdam: Elsevier Science, 13-25, 2000. [22] M. T. Turvey and R. E. Shaw: Ecological foundations of cognition, in R. Nunez and W. J. Freeman (eds), Reclaiming Cognition: The Primacy of Action, Intention and Emotion, United Kingdom: Imprint Academic, 95-110, 2000.

[23] A. M. Young: The Geometry of Meaning, Mill Valley, CA: Robert Briggs

Associates, 1976.

[24] L.A. Zadeh: Outline of a New Approach to the Analysis of Complex Systems and Decision Processes, IEEE Transactions on Systems, Man and Cybernetics, SMC-3, 28-44, 1973. 